\title{
Erratum to "Muscular Contraction Ability Develops in the Lower Trapezius Muscle of the Dominant Arm in Team Hand-Ball Players" [Advances in Bioscience and Biotechnology 6 (2015) 368-374]
}

\author{
Hajime Fujimoto1,2, Tamotsu Yabumoto², Hiroyuki Sugimori², Sohee Shin'2, \\ Tsuneo Watanabe ${ }^{2}$, Toshio Matsuoka ${ }^{2}$ \\ ${ }^{1}$ Faculty of Health and Sport Sciences, University of Tsukuba, Tsukuba, Japan \\ ${ }^{2}$ Department of Sports Medicine and Sports Science, Gifu University School of Medicine, Gifu, Japan \\ Email: yabumoto@gifu-u.ac.jp
}

Received 24 April 2015; accepted 17 May 2015; published 20 May 2015

Copyright (C) 2014 by authors and Scientific Research Publishing Inc.

This work is licensed under the Creative Commons Attribution International License (CC BY).

http://creativecommons.org/licenses/by/4.0/

(c) (i) Open Access

The original online version of this article (Fujimoto, H., et al. (2015) Muscular Contraction Ability Develops in the Lower Trapezius Muscle of the Dominant Arm in Team Hand-Ball Players. Advances in Bioscience and Biotechnology, 6, 368-374. http://dx.doi.org/10.4236/abb.2015.65036) was mistaken of the mean value in Table 1 , we revised Table 1 . The authors wish to correct the errors to:

Table 1

\begin{tabular}{cccccccc}
\hline & Age & Height $(\mathrm{cm})$ & Weight $(\mathrm{kg})$ & Body fat (\%) & BMI & Competitive experience (year) & Dominant arm \\
\hline 1 & 19 & 173 & 71.8 & 16.3 & 24.0 & 5 & $\mathrm{Rt}$ \\
2 & 19 & 174 & 71.6 & 17.6 & 23.6 & 5 & $\mathrm{Rt}$ \\
3 & 20 & 174 & 59.1 & 10.9 & 19.5 & 6 & $\mathrm{Lt}$ \\
4 & 20 & 183 & 76.6 & 16.9 & 22.9 & 6 & $\mathrm{Rt}$ \\
5 & 20 & 182 & 68.0 & 13.3 & 20.5 & 6 & $\mathrm{Rt}$ \\
6 & 22 & 171 & 70.3 & 18.8 & 24.0 & 8 & $\mathrm{Rt}$ \\
7 & 22 & 176 & 66.9 & 14.3 & 21.6 & 8 & $\mathrm{Rt}$ \\
8 & 22 & 177 & 68.3 & 15.8 & 21.8 & 8 & $\mathrm{Rt}$ \\
\hline
\end{tabular}


H. Fujimoto et al.

\begin{tabular}{cccccccc}
\multicolumn{2}{l}{ Continued } \\
\hline 9 & 20 & 170 & 64.4 & 17.3 & 22.3 & 6 & $\mathrm{Rt}$ \\
10 & 20 & 175 & 66.3 & 16 & 21.6 & 6 & $\mathrm{Rt}$ \\
11 & 22 & 160 & 60.7 & 15.8 & 23.7 & 8 & $\mathrm{Rt}$ \\
Mean & 20.5 & 174.1 & 67.6 & 15.7 & 22.3 & 6.5 & 1.2 \\
SD & 1.2 & 6.2 & 5.0 & 2.2 & 1.5 & & \\
\hline
\end{tabular}

